\title{
Fattori influenti sul ricorso ad Internet nei processi gestionali delle micro-imprese ${ }^{\bullet}$
}

\author{
GIAN LUCA GREGORI* SILVIO CARDINALI** \\ ChIARA GigLIARANO*** FEDERICA PASCUCCI ${ }^{* * * *}$
}

\begin{abstract}
Obiettivo del paper: Il presente lavoro intende offrire un contributo di conoscenza sul rapporto tra piccole imprese e Internet, analizzando in particolare $i$ principali fattori influenti sulla decisione di utilizzare tale tecnologia nei processi di management da parte delle micro-imprese artigiane.

Metodologia: Dopo un'analisi della letteratura, è stato costruito un modello di ricerca e sono state elaborate quattro ipotesi (sottoposte successivamente ad una verifica empirica su un campione di 600 imprese marchigiane), relative ad altrettanti fattori, consideranti rilevanti in letteratura ai fini del ricorso o meno ad Internet: dimensione aziendale, localizzazione geografica, settore economico di appartenenza e ampiezza del mercato di vendita dell'impresa.

Risultati: Dall'analisi statistica svolta è emerso che la diffusione di Internet nelle imprese indagate è ancora piuttosto bassa, e che la decisione di utilizzare tale tecnologia è influenzata dalla dimensione dell'impresa e dal settore, ma non dalla localizzazione.

Limiti della ricerca: I risultati presentati costituiscono la prima fase di un processo di ricerca più ampio, volto ad indagare anche le modalità di utilizzo di Internet e le connesse implicazioni per la competitività delle imprese.

Implicazioni pratiche: Il paper evidenzia i limiti dell'adozione di Internet nelle microimprese, cercando di individuarne le cause, dalle quali possono derivare utili indicazioni manageriali per il miglioramento della competitività aziendale.
\end{abstract}

- Pur essendo frutto di un lavoro comune, i paragrafi possono essere attribuiti nel modo seguente: n. 1 a Gian Luca Gregori, n. 2 a Federica Pascucci, n. 3 a Silvio Cardinali, n. 4 a Chiara Gigliarano e n. 5 congiuntamente a Gian Luca Gregori, Silvio Cardinali e Federica Pascucci.

* $\quad$ Ordinario di Economia e Gestione delle Imprese - Università Politecnica delle Marche e-mail: g.gregori@univpm.it

** Ricercatore di Economia e Gestione delle Imprese - Università Politecnica delle Marche e-mail: s.cardinali@univpm.it

*** Ricercatore di Statistica - Università Politecnica delle Marche e-mail: c.gigliarano@univpm.it

**** Ricercatore di Economia e Gestione delle Imprese - Università Politecnica delle Marche e-mail: f.pascucci@univpm.it 
Originalità del lavoro: Pochi sono gli studi aventi per oggetto le micro-imprese artigiane, che pure rivestono un'importanza economica rilevante nel nostro Paese e in Europa; il presente lavoro intende contribuire a colmare tale gap, per comprendere le determinanti e le implicazioni di Internet in questa particolare tipologia di imprese.

Parole chiave: PMI; micro-imprese; Internet; dimensione aziendale; internazionalizzazione

Purpose of the paper: This paper aims to expand on the knowledge regarding the relationship between small businesses and Internet, and in particular it wants to analyze the main factors that influence the decision to use this technology.

Methodology: After a thorough analysis of the literature, a model was created and four hypotheses were developed (which were subsequently subjected to empirical testing on a sample of 600 firms), These are related to different factors that are relevant to the adoption of the Internet: company size, geographical location, the type of industry and the level of internationalization.

Findings: Statistical analysis carried out shows that the use of the Internet in the companies surveyed is still not so widespread, and that the decision to use this technology is influenced by the size of the company and the sector it operates in, while location is not a significant factor.

Limitations of research: The results of the research constitute the first phase of a larger research process. This research process also investigates the ways in which the Internet is used and related implications involved in the competitiveness of enterprises.

Practical implications: The paper highlights the limitations in the adoption of the Internet in micro-enterprises, and it tries to identify the causes of this situation. From these elements useful information can be derived for business management in terms of improving firm competitiveness.

Originality of the paper: Few studies on this topic have addressed the micro-enterprise sector, which among other sectors also holds a significant economic role in Italy and in Europe. This paper aims to fill this gap in order to understand the determinants and implications of the Internet for this particular type of companies.

Key words: SMEs; micro-enterprises; Internet; company size; internationalization

\section{Introduzione}

Numerose ricerche evidenziano che le imprese attive online crescono più in fretta e sono maggiormente internazionalizzate dal lato delle vendite (The Boston Consulting Group, 2011, p. 16); tuttavia, secondo recenti studi, la penetrazione di Internet tra le piccole imprese in Italia è ancora piuttosto bassa, nonostante i vantaggi che queste possono trarre dall'utilizzo della Rete nei propri processi di business, in termini di produttività e di crescita; è stato infatti rilevato che Internet potrebbe costituire un'innovazione importante e strategica per molte imprese minori: la flessibilità organizzativa e la rapidità decisionale, derivanti dalla ridotta dimensione, renderebbero infatti queste imprese più predisposte di quelle grandi a sfruttare la dinamicità e la varietà offerta dagli ambienti elettronici, mediante la sperimentazione di nuovi modelli di business e l'innovazione delle attività (Pascucci, 2012, p. 177; Jutla et al., 2002, p. 140). Esistono studi empirici che 
mostrano una relazione positiva significativa tra l'adozione di Internet e la performance economico-finanziaria delle PMI, misurata in termini di aumento delle entrate e riduzione dei costi, anche se con alcune differenze in funzione del settore di appartenenza (Johnston et al., 2007, p. 359; Drennan e McColl-Kennedy, 2003, p. 306; Davis e Harveston, 2000, p. 115).

Le nuove tecnologie dell'informazione e delle telecomunicazioni potrebbero fornire un contributo significativo al miglioramento della competitività delle PMI italiane, anche a livello internazionale; non può sfuggire infatti l'importante ruolo giocato dalle PMI nell'economia nazionale ed europea: esse costituiscono il 99,8\% delle imprese dell'UE a 27 Paesi e il 67,4\% dell'occupazione totale ("EU Smes in 2012: at the crossroads", Rapporto Annuale sulle PMI in Europa, settembre 2012).

Alla luce della rilevanza che tale categoria riveste, appare abbastanza sorprendente la carenza di studi sull'utilizzo di Internet da parte di queste imprese; al fine di colmare tale gap di conoscenza, il presente lavoro si propone di analizzare lo stato dell'adozione di Internet tra le micro e piccole imprese marchigiane a carattere artigianale, e i fattori influenti su tale adozione. Si noti che le Marche sono una regione a "forte vocazione artigiana": misurata come quota di imprese artigiane sul totale imprese, questa risultava pari al 34,8\% (in termini assoluti si tratta di quasi 46mila imprese) nel 2009 (Centro Studi CNA, 2012).

Il framework e le ipotesi della ricerca sono stati costruiti sulla base dell'analisi della letteratura riguardante l'adozione delle innovazioni (in particolare, ICT e Internet) nelle PMI, e successivamente sottoposti a verifica empirica su un campione di 600 imprese marchigiane. Si tratta di una prima fase di un processo di ricerca più ampio, il quale prevede l'analisi dei fattori che influiscono sull'adozione di Internet nelle microimprese e delle modalità di utilizzo dello strumento anche a fini di marketing.

La struttura del lavoro è la seguente: innanzitutto verrà sinteticamente presentata l'analisi della letteratura riguardante il rapporto tra Internet e PMI; successivamente verranno illustrati il modello, le ipotesi e la metodologia di ricerca; nei paragrafi finali verranno descritti i risultati e discusse le principali implicazioni derivanti dall'analisi svolta, insieme alle limitazioni dello studio e alle prospettive di ricerca future.

\section{Internet e PMI: una review della letteratura}

Il rapporto tra PMI e innovazione tecnologica, considerando in modo particolare quella legata all'ICT e ad Internet, è uno dei temi più investigati dagli studiosi di management, oltre che dagli economisti industriali.

La maggior parte degli studi però riguardano l'ampio ed eterogeneo comparto delle PMI (Cedrola, 2009; Alam, 2009; Levy et al., 2005, p. 6; Grandon e Pearson, 2004; Drew, 2003; Vescovi e Ieseppon, 2002, p. 511), che comprende al suo interno organizzazioni di varia dimensione (da 1 a 250 addetti) e struttura.

La dimensione aziendale costituisce un fattore significativo nell'influenzare il comportamento delle PMI nei confronti delle tecnologie (ICT e Internet) e quindi 
nella decisione di adottare o meno l'innovazione. La relazione tra dimensione aziendale e adozione di Internet può essere spiegata secondo due prospettive differenti:

a) la dimensione come proxy della disponibilità di risorse da investire; secondo tale prospettiva la minore dimensione rappresenta un ostacolo all'adozione della tecnologia, in quanto indicatore della carenza di risorse finanziarie, gestionali e di conoscenza, la quale caratterizza le imprese più piccole.

b) La dimensione come proxy della "complessità informativa" all'interno della struttura organizzativa aziendale; secondo tale prospettiva le imprese di minore dimensione non sentono la necessità di adottare Internet poiché devono gestire un livello di complessità informativa ridotto, rispetto alle imprese di dimensione maggiore; via via che l'organizzazione cresce, aumenta la complessità da gestire dal punto di vista informativo e quindi lo stimolo ad investire nelle tecnologie Internet.

Alcuni studiosi hanno parlato in proposito dell'esistenza di un "digital divide" anche all'interno delle PMI, oltre che tra queste ultime e le grandi imprese (Arbore e Ordanini, 2006, p. 84). Secondo Levenburg (2005, p. 101), non soltanto esistono significative differenze tra micro, piccole e medie imprese sulle modalità di utilizzo delle tecnologie, nel senso che le micro-imprese sono più in ritardo e mostrano un utilizzo più elementare, ma anche nell'entità dei benefici conseguenti: quando le imprese minori iniziano ad utilizzare Internet in modo più strutturato i benefici che ne traggono sono più pronunciati rispetto alle altre categorie di imprese.

Alla luce di tali studi, indagare il rapporto tra Internet e PMI senza considerare l'eterogeneità dimensionale può costituire un limite, la cui conseguenza sarebbe l'inconsistenza e la contraddittorietà nei risultati raggiunti dalle ricerche (Bordonaba-Juste et al., 2012, p. 213; Parker e Castleman, 2007, p. 30; Bengtsson et al., 2007, p. 38; Levenburg, 2005, p. 101).

Il presente lavoro si inserisce in tale filone di ricerca, riconoscendo, da un lato, la rilevanza della dimensione aziendale anche all'interno della categoria delle PMI e, dall'altro, l'opportunità di focalizzare l'attenzione su una parte specifica di tale comparto, sovente trascurata dagli studi ovvero quella delle micro-imprese ${ }^{1}$ ed in particolare artigiane ${ }^{2}$. Dall'analisi della letteratura, sia italiana sia estera, emerge

1 Si noti che la categoria delle microimprese è stata introdotta soltanto nel 2003 con la raccomandazione della Commissione Europea n.361 del 6 maggio, nella quale sono stati definiti i parametri in termini di numero di addetti e di fatturato, necessari per classificare le imprese in funzione della loro dimensione. Si può definire microimpresa, una che occupa fino a 10 addetti, che fattura annualmente non più di 2 milioni di euro e che possiede un totale di bilancio al di sotto dei 2 milioni di euro.

2 È artigiana l'impresa che ha come scopo prevalente lo svolgimento di un'attività di produzione di beni, anche semilavorati, o di prestazioni di servizi, escluse le attività agricole, commerciali, di intermediazione di beni o ausiliare di queste ultime, di somministrazione di alimenti o di bevande. Il numero di occupati nell'impresa artigiana è soggetto a limitazioni, differenti a seconda del tipo di attività svolta, ma con un massimo di 40 (Legge 443 del 1985 - Legge Quadro sull'Artigianato). 
infatti un gap di conoscenza significativo rispetto all'utilizzo di Internet da parte delle imprese di minore dimensione ed in particolare, delle micro-imprese: poco è stato pubblicato sul comportamento tecnologico di questa peculiare tipologia di imprese (Bordonaba-Juste et al., 2012, p. 213; Davis e Vladica, 2006, p. 1; Fillis et al., 2004, p. 180). In alcune indagini vengono esplicitamente escluse dal campione analizzato le imprese più piccole (Bayo-Moriones e Lera-Lopez, 2007, p. 357; Ihlstrom e Nilsson, 2003, p. 212; Wade et al., 2004, p. 343).

Le microimprese presentano alcuni tratti distintivi, che non possono non influire sulle scelte di adozione delle innovazioni; essi sono i seguenti:

- la limitata organizzazione aziendale, con suddivisione dei ruoli tra il personale, visto che in non pochi casi è lo stesso imprenditore a ricoprire ruoli diversi e ad esercitare un'influenza dominante su tutte le scelte aziendali;

- la natura sovente familiare dell'impresa e quindi la sostanziale coincidenza tra il bilancio aziendale e quello familiare;

- l'ampio ricorso all'autofinanziamento quale modalità di reperimento delle risorse finanziarie;

- la flessibilità, intesa come capacità di adattarsi ai cambiamenti indotti dall'evoluzione del contesto esterno;

- la profonda conoscenza della propria clientela, per lo meno di quella più importante, con la quale si sviluppano rapporti anche personali e diretti.

Dalla review della letteratura emergono i due seguenti filoni di ricerca principali riguardanti le imprese minori.

1. L'analisi dei fattori (interni ed esterni all'organizzazione aziendale), che impediscono o che agevolano l'adozione di Internet nei processi di management; Hairuddin et al. (2012, p. 497) hanno investigato le barriere all'adozione delle tecnologie dell'informazione presso le microimprese della Malaysia, produttrici dei tradizionali "batik": mancanza di conoscenze e di capacità e scarsità di risorse finanziarie sono i principali ostacoli riscontrati. Anche le caratteristiche del settore di appartenenza sono state considerate tra $\mathrm{i}$ fattori influenti, in particolare l'intensità della concorrenza (Peltier et al., 2012, p. 423; Pontikakis et al., 2006, p. 346; Dholakia e Kshetri, 2004, p. 319; Kowtha e Choon, 2001, p. 237; Hollestein, 2004, p. 338; Premkumar e Roberts, 1999, p. 481; Premkumar e Ramamurthy, 1995, p. 334), il livello di incertezza (Peltier et al., 2012, p. 423), il tipo di attività svolta (Cheung e Huang, 2002, p. 382), la pressione competitiva internazionale (Hollestein, 2004, p. 338), le pressioni ricevute da clienti e da fornitori ad adottare le nuove tecnologie ${ }^{3}$. Secondo i risultati di questi studi, nei settori caratterizzati da una maggiore intensità della concorrenza e da un maggior livello di incertezza le imprese mostrano una superiore propensione ad adottare nuove tecnologie. Arbore e Ordanini (2006, p. 90) hanno trovato una relazione

3 Le PMI nei settori high tech e knowledge intensive sono maggiormente propense all'adozione di Internet e ne fanno un maggiore uso rispetto alle PMI dei settori manifatturieri tradizionali e dei servizi; fra queste ultime due categorie non sono state rilevate differenze significative (Drew, 2003, p. 86). 
significativa tra la grandezza della città nella quale l'impresa è localizzata e l'adozione di una connessione Internet a banda larga: specie per le imprese di minore dimensione infatti la localizzazione in piccoli centri (ad esempio, nelle aree rurali) può rappresentare un ostacolo, in quanto minori sono le risorse a disposizione delle stesse per adottare ed utilizzare in modo efficace l'innovazione (ad esempio, meno disponibilità di servizi specializzati o fornitori di IT).

2. L'analisi delle implicazioni che l'adozione di Internet può produrre sulla performance aziendale. Pur essendo consapevoli della complessità del rapporto tra adozione della tecnologia e performance ${ }^{4}$, va rilevato che nella letteratura di marketing non pochi studi hanno evidenziato una relazione positiva. Poon e Swatman (1999, p. 11), mediante un'indagine qualitativa su 23 imprese australiane, hanno analizzato i benefici percepiti derivanti dall'attività online ed in particolare dell'e-commerce, distinguendoli in "diretti" ed "indiretti", di "breve" e di "lungo termine". Tra i benefici Walcuzuch et al. (2000, p. 565) hanno rilevato quelli relativi all'internazionalizzazione: le piccole imprese olandesi (con meno di 50 dipendenti) percepiscono Internet come un utile canale per l'internazionalizzazione, in quanto consente di abbattere le distanze geografiche e comunicare in tutto il mondo.

\section{Ipotesi di ricerca e metodologia dell'indagine}

Obiettivo del paper è di studiare la relazione tra imprese di piccola dimensione e utilizzo di Internet, al fine di verificare, non soltanto il livello di adozione di questa tecnologia, ma anche i fattori maggiormente influenti sull'adozione.

La figura n.1 presenta il framework dello studio e le connesse ipotesi di ricerca, distinguendo tra la prima fase del progetto, i cui risultati sono presentati in questo lavoro, e la seconda fase, che è ancora in corso di svolgimento. Mentre nella prima fase l'oggetto di ricerca si riferisce alla decisione strategica dell'organo di governo dell'impresa di adottare Internet, nella seconda fase l'attenzione si sposterà sull'azione gestionale, ovvero sulle modalità mediante le quali Internet viene utilizzata nei processi di marketing. Si tratta di due differenti, seppur interrelati, piani di analisi, riconducibili alla distinzione tra governo e gestione dell'impresa, nell'ottica dell'approccio sistemico-vitale (Golinelli, 2009, p. XII; Golinelli e Gatti, 2007, p. 23).

Il modello cerca di descrivere il ricorso ad Internet nei processi di management da parte delle microimprese artigiane marchigiane come una funzione delle seguenti quattro variabili ${ }^{5}$.

4 Si pensi, ad esempio, alle ricerche di Brynjolfsson (1993) aventi per oggetto le implicazioni delle tecnologie informatiche sulla produttività.

5 Per "adozione di Internet" nel presente lavoro si è inteso l'utilizzo della Rete da parte dell'impresa nell'ambito della propria attività gestionale. 
Fig. 1: Modello di ricerca e ipotesi

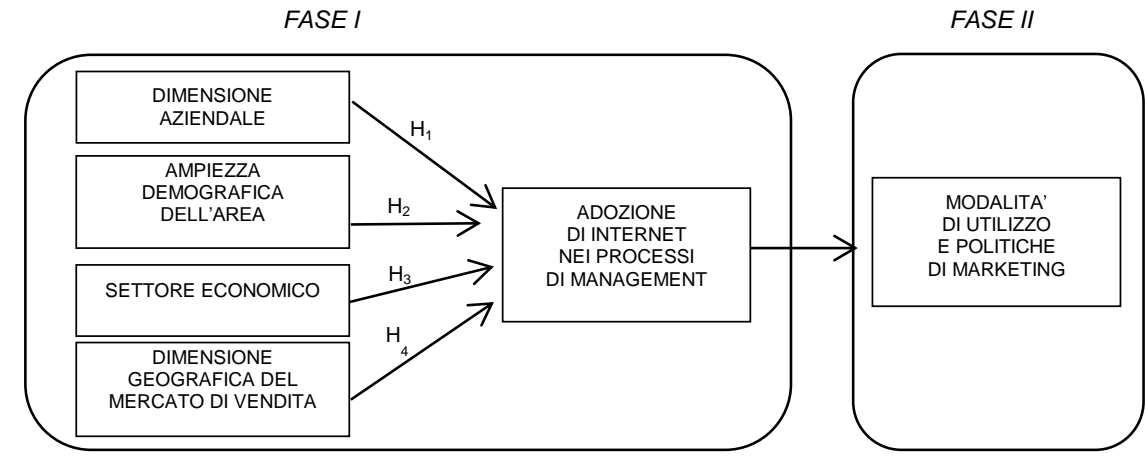

Fonte: ns. elaborazione

1. La dimensione aziendale, quale espressione della disponibilità di risorse e di competenze interne all'organizzazione che possono essere impiegate per un'efficace adozione della tecnologia. I risultati degli studi aventi per oggetto la relazione tra dimensione aziendale e adozione di Internet (così come delle ICT) mostrano esiti contrastanti: alcuni hanno verificato l'esistenza di una relazione positiva (Bordonaba-Juste et al., 2012, p. 220; Kowtha e Choon, 2001, p. 237; Khemthong e Roberts, 2006; Lertwongsatien e Wongpinunwatana, 2003; Dholakia e Kshetri, 2004, p. 319; Khemthong e Roberts, 2006, 56; Burke, 2005, p. 83; Levenburg, 2005, p. 101; Arbore e Ordanini, 2006, p. 89; Dandridge e Levenburg, 2000, p. 86; Thong e Yap, 1995, p. 437), mentre altri non hanno trovato nessuna relazione significativa (Tan et al., 2010, p. 50; Pontikakis et al., 2005, p. 349). Tale eterogeneità dei risultati induce la necessità di indagare ulteriormente il ruolo della dimensione aziendale, pertanto la prima ipotesi di ricerca è la seguente:

$H_{1} \quad$ L'adozione di Internet è positivamente influenzata dalla dimensione aziendale.

2. La localizzazione geografica dell'impresa, quale espressione della disponibilità di risorse ambientali, dunque "esterne" all'impresa, che può agevolare o meno l'adozione di Internet. Poiché difficilmente le imprese di piccola dimensione riescono a gestire autonomamente con proprie risorse interne la complessità connessa all'inserimento delle nuove tecnologie, dal punto di vista sia strettamente tecnico, sia strategico, l'ampiezza e la qualità dell'offerta di servizi avanzati alle imprese può costituire un fattore determinante nell'adozione di Internet. A seconda di dove l'organizzazione è localizzata, tale offerta di servizi può variare in modo significativo: la dotazione di servizi e di infrastrutture è normalmente maggiore nei grandi centri urbani rispetto ai piccoli centri o alle aree rurali, nella costa rispetto alle aree dell'entroterra. Da ciò deriva la seguente ipotesi di ricerca: 


\section{$\mathrm{H}_{2} \mathrm{~L}$ 'adozione di Internet è influenzata positivamente dall'ampiezza demografica dell'area nella quale l'impresa è localizzata.}

3. Il settore economico di appartenenza dell'impresa, quale espressione del tipo di attività posta in essere dall'organizzazione. Riguardo all'influenza che il settore può esercitare sulla propensione delle imprese ad adottare Internet, i risultati ai quali sono pervenuti gli studiosi non sono univoci: secondo alcuni l'adozione di Internet (e dell'ICT in generale) è influenzata dalle caratteristiche del settore di appartenenza dell'impresa (Love et al., 2005, p. 961), secondo altri non vi sarebbe alcuna influenza significativa (Thong e Yap, 1995, p. 436; BordonabaJuste et al., 2012, p. 221; Pontikakis et al., 2005, p. 349) mentre Bengtsson et al. (2007, p. 39) hanno verificato che la pressione del mercato ha un'influenza soltanto marginale sull'adozione di Internet da parte delle PMI di ogni dimensione. Ne deriva che l'influenza del settore deve essere ulteriormente indagata. La terza ipotesi di ricerca è pertanto la seguente:

$\mathrm{H}_{3}$ L'adozione di Internet è influenzata dal settore economico nel quale l'impresa opera.

4. La dimensione geografica del mercato di vendita dei propri prodotti, che per non poche piccole imprese è molto ristretto (livello regionale, se non addirittura provinciale), per altre più ampio, fino a diventare internazionale. Relativamente al livello di internazionalizzazione dell'impresa, è possibile evidenziare che i contributi della letteratura interpretano la presenza nei mercati internazionali, in alcuni casi, come causa (Bayo-Moriones e Lera-Lopez, 2007) e, in altri, come conseguenza del ricorso e meno alle nuove tecnologie dell'informazione (Mostafa et al., 2005). In questa fase della ricerca si è voluto indagare l'internazionalizzazione come fattore causa, sottoponendo a verifica la seguente ipotesi:

\section{$\mathrm{H}_{4} \mathrm{~L}$ 'adozione di Internet è influenzata dalla dimensione locale, nazionale e internazionale del mercato di vendita dell'impresa.}

Le ipotesi di ricerca sono state sottoposte a verifica empirica nel corso del 2012 su un campione di 600 imprese marchigiane, alle quali è stato somministrato un questionario strutturato mediante intervista telefonica. Il campione è stato costruito dall'Ente Bilaterale Artigianato Marche (EBAM), ricorrendo ad un campionamento stratificato (sulla base delle variabili di settore, fatturato e dimensione) delle imprese marchigiane appartenenti all'archivio Asia prodotto dall'Istat.

I grafici seguenti riportano alcune informazioni preliminari inerenti il campione su cui è stata condotta l'analisi. In particolare, la figura n.2 illustra la distribuzione delle imprese in funzione della dimensione, ovvero del numero di addetti. Il $35 \%$ delle imprese artigiane esaminate è costituito da un solo addetto, mentre il $38 \%$ di esse non supera 5 addetti. Pertanto, la maggioranza di esse rientra nella categoria della micro-impresa.

Come illustrato nella figura n. 3, il 29\% delle imprese appartenenti al campione oggetto di studio è localizzato nella provincia di Ancona, seguito da un $26 \%$ di 
imprese che hanno sede in provincia di Macerata e un 23\% in provincia di PesaroUrbino; seguono le imprese della provincia di Ascoli Piceno (13\%) e di Fermo (9\%).

Fig. 2: Distribuzione delle imprese per numero di addetti

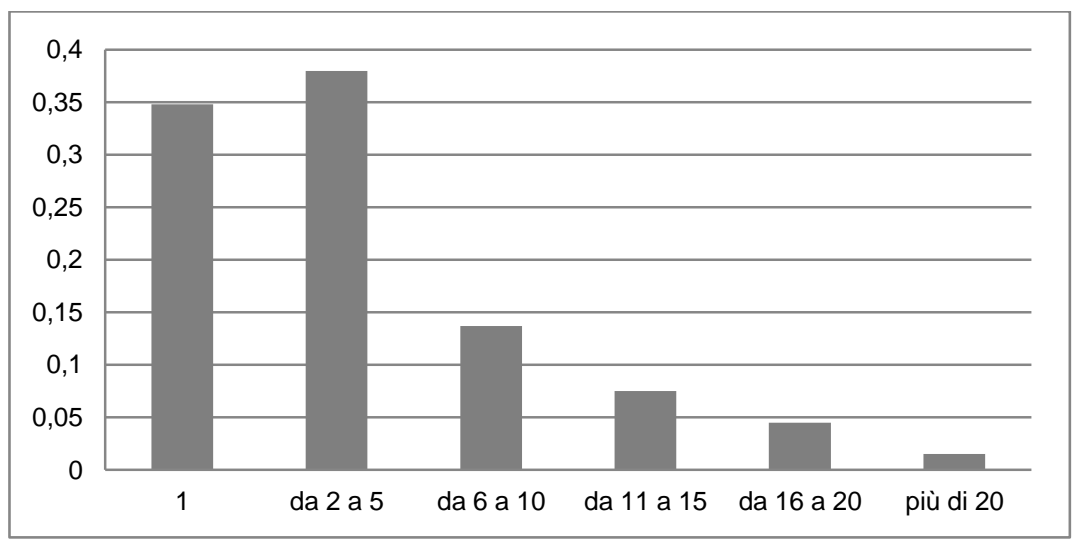

Fonte: ns. elaborazione su dati EBAM

Fig.3: Distribuzione delle imprese per provincia

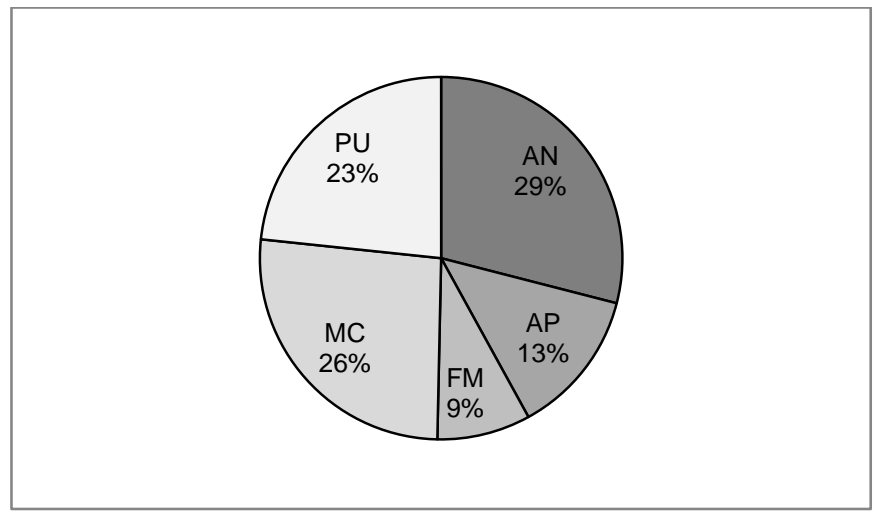

Fonte: ns. elaborazione su dati EBAM

Come si può notare dalla figura n.4, la distribuzione delle imprese artigiane è piuttosto omogenea tra i diversi settori di attività.

Al fine di testare la significatività dell'impatto dei fattori indicati nella figura n.1 sull'utilizzo di Internet da parte delle imprese, è stato sviluppato un modello di regressione logistica, in cui la variabile dipendente è rappresentata dalla probabilità di utilizzare Internet $(y=1)$ rispetto alla probabilità di non utilizzarlo $(y=0)$. Com'è 
noto, il modello di regressione logistica è un modello di regressione, applicato nei casi in cui la variabile dipendente $y$ sia di tipo dicotomico, come nel caso in esame.

Fig. 4: Distribuzione delle imprese per settore d'attività

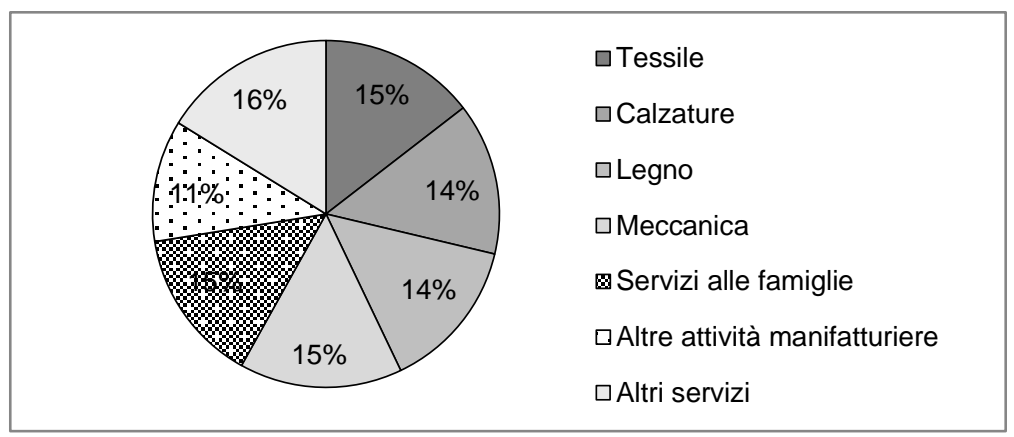

Fonte: ns. elaborazione su dati EBAM

Come variabili esplicative sono state considerate il numero di addetti (come proxy della dimensione aziendale), la dimensione e la vicinanza alla costa del comune nel quale l'impresa è localizzata e la provincia (come proxy della localizzazione geografica dell'impresa), il codice ATECO di riferimento dell'attività svolta dall'impresa (come proxy del settore economico), e la percentuale del fatturato realizzato a livello regionale, nazionale ed internazionale (come proxy della dimensione geografica del mercato di vendita).

\section{Principali risultati ottenuti}

Dall'indagine emerge innanzitutto che nel 2012 meno di un terzo $(31.17 \%)$ delle imprese artigiane utilizza Internet per la propria attività, ed il restante $68.83 \%$ dichiara di non usufruirne.

Il numero medio di addetti delle imprese artigiane che non utilizzano Internet è di 3.4 , mentre in media sono 7.4 gli addetti delle imprese propense a navigare in Rete.

Dunque, sembra che la dimensione aziendale influenzi notevolmente la propensione ad utilizzare Internet da parte delle imprese artigiane marchigiane.

Le Tabelle 1-4 riportano ulteriori statistiche descrittive.

Come si può notare dalla Tab. 1, i principali settori in cui si utilizza Internet sono il settore meccanico e quello della lavorazione del legno, mentre i servizi alle famiglie non ricorrono quasi per niente alla navigazione sul web.

La Tab. 2 mostra inoltre che nelle provincie di Fermo e di Ascoli Piceno l'incidenza di imprese artigiane, che usano Internet è superiore alla media regionale. 
Tab. 1: Propensione (\%) all'uso di Internet per settore di attività economica ${ }^{6}$

\begin{tabular}{|l|r|r|r|r|r|r|r|r|}
\hline & & & & Altre attività & Servizi alle & & \\
Internet & Tessile & Calzature & Legno & Meccanica & manifatturiere & famiglie & Altri servizi & Totale \\
\hline 0 & 68.78 & 67.06 & 41.18 & 42.86 & 64.71 & 96.51 & 89.69 & 68.83 \\
1 & 31.22 & 32.94 & 58.82 & 57.14 & 35.29 & 3.49 & 10.31 & 31.17 \\
Totale & 100 & 100 & 100 & 100 & 100 & 100 & 100 & 100 \\
\hline
\end{tabular}

Fonte: ns. elaborazioni su dati EBAM

Non si rilevano invece differenze evidenti nella propensione all'uso di Internet tra comuni litoranei e non, come mostrato nella Tabella n. 3. La medesima tabella mostra inoltre che la frequenza di imprese artigiane utilizzatrici di Internet è pressoché la stessa, indipendentemente dal fatto che l'impresa abbia sede in un comune il cui numero di abitanti è inferiore al numero mediano di abitanti nelle Marche (circa 8600 abitanti) oppure in un comune la cui dimensione supera la mediana regionale.

Tab. 2: Propensione (\%) all'uso di Internet per provincia

\begin{tabular}{|l|r|r|r|r|r|r|}
\hline Internet & Ancona & Ascoli Piceno & Fermo & Macerata & Pesaro Urbino & \multicolumn{1}{c|}{ Totale } \\
\hline 0 & 70.69 & 64.1 & 58.00 & 75.95 & 65.00 & 68.83 \\
1 & 29.31 & 35.9 & 42.00 & 24.05 & 35.00 & 31.17 \\
Totale & 100 & 100 & 100 & 100 & 100 & 100 \\
\hline
\end{tabular}

Fonte: ns. elaborazioni su dati EBAM

Tab. 3: Propensione (\%) all'uso di Internet per tipologia del Comune

\begin{tabular}{|l|rr|rr|r|}
\hline Internet & \multicolumn{2}{|c|}{ Comune litoraneo } & \multicolumn{2}{|c|}{ Numero abitanti del Comune } & Totale \\
& NO & Sì & $<8600$ & $>8600$ & \\
\hline 0 & 67.61 & 72.16 & 67.58 & 69.97 & 68.83 \\
1 & 32.39 & 27.84 & 32.42 & 30.03 & 31.17 \\
Totale & 100 & 100 & 100 & 100 & 100 \\
\hline
\end{tabular}

Fonte: ns. elaborazioni su dati EBAM

Infine, dalla Tab. 4 si può notare come l'ampiezza del mercato di vendita delle imprese artigiane influenzi notevolmente l'uso di Internet. Infatti, solo il 16.1\% delle imprese che vendono i loro prodotti esclusivamente all'interno della provincia usa Internet, mentre tale percentuale aumenta se l'impresa vende su tutto il territorio regionale $(27.2 \%)$ e nazionale $(55.5 \%)$. Esportazioni europee ed extra-europee influenzano in maniera ancora più rilevante il ricorso ad Internet; in particolare,

6 In "altre attività Manifatturiere" sono considerate tutte le attività non ricomprese nelle precedenti, fra cui le attività agroindustriali e l'industria chimica; restano invece escluse le attività del settore delle costruzioni. In merito alla categoria "altri servizi" sono ricompresi i servizi alle imprese, alla collettività, e quelli di natura anche commerciale. 
1'81.5\% delle imprese che esportano i loro prodotti in Europa e il 60\% di quelle che commerciano anche al di fuori dell'Europa utilizza Internet per le proprie attività.

Tab. 4: Propensione (\%) all'uso di Internet per ampiezza del mercato di vendita

\begin{tabular}{|l|rr|rr|rr|rr|rr|r|r|}
\hline \multirow{2}{*}{ Internet } & \multicolumn{2}{|c|}{ Al più nella Provincia } & \multicolumn{2}{|c|}{ Al più nella Regione } & \multicolumn{2}{|c|}{ Al più in Italia } & \multicolumn{2}{|c|}{ Al più in Europa } & \multicolumn{2}{|c|}{ Anche Extra Europa } & \multicolumn{2}{|c|}{ Totale } \\
& NO & Sì & NO & Sì & NO & Sì & NO & Sì & NO & Sì & \\
\hline 0 & 51.1 & 84.0 & 68.1 & 72.8 & 76.0 & 44.5 & 71.2 & 18.5 & 69.8 & 40.0 & 68.8 \\
1 & 48.9 & 16.1 & 31.9 & 27.2 & 24.0 & 55.5 & 28.8 & 81.5 & 30.2 & 60.0 & 31.2 \\
Totale & 100 & 100 & 100 & 100 & 100 & 100 & 100 & 100 & 100 & 100 & 100 \\
\hline
\end{tabular}

Fonte: ns. elaborazioni su dati EBAM

Prima di procedere con l'implementazione del modello di regressione logistica, per analizzare i legami di dipendenza esistenti tra le diverse variabili esplicative è stato effettuato il test chi-quadrato di Pearson per la verifica dell'indipendenza tra le variabili nominali (dimensione del comune, comune litoraneo, provincia, export e settore). Inoltre, per verificare l'indipendenza in media tra la variabile continua (addetti totali) e le variabili nominali si è ricorso al test $t$ di Student e all'analisi della varianza (ANOVA), i cui risultati sono riportati nella Tabella 5.

Tab. 5: Valori delle statistiche test per l'indipendenza tra le variabili esplicative ( $p$-value tra parentesi)

\begin{tabular}{|c|c|c|c|c|c|}
\hline & $\begin{array}{l}\text { Dimensione } \\
\text { del Comune }\end{array}$ & $\begin{array}{l}\text { Comune } \\
\text { litoraneo }\end{array}$ & Provincia & Export & Settore \\
\hline \multicolumn{6}{|l|}{$\begin{array}{l}\text { Dimensione del } \\
\text { Comune }\end{array}$} \\
\hline Comune litoraneo & $\begin{array}{l}148.55 \\
(0.00)^{1}\end{array}$ & & & & \\
\hline Provincia & $7.58(0.11)^{1}$ & $29.34(0.00)^{1}$ & & & \\
\hline Export & $3.28(0.51)^{1}$ & $6.06(0.19)^{1}$ & $\begin{array}{l}26.01 \\
(0.05)^{1}\end{array}$ & & \\
\hline Settore & $10.61(0.10)^{1}$ & $7.71(0.26)^{1}$ & $\begin{array}{l}252.27 \\
(0.00)^{1}\end{array}$ & $\begin{array}{l}192.06 \\
(0.00)^{1}\end{array}$ & \\
\hline Addetti Totali & $-0.06(0.96)^{2}$ & $2.83(0.01)^{2}$ & $1.83(0.12)^{3}$ & $\begin{array}{l}34.76 \\
(0.00)^{3}\end{array}$ & $\begin{array}{l}16.02 \\
(0.00)^{3}\end{array}$ \\
\hline
\end{tabular}

Dalla Tabella 5 emerge che la maggior parte delle coppie di variabili esplicative sono indipendenti. La variabile Comune litoraneo presenta, invece, una dipendenza significativa con la dimensione del comune, con la provincia e con il numero di addetti totali. La variabile settore ha una dipendenza significativa con la provincia, con le esportazioni e con il numero di addetti totali. Infine, esiste una dipendenza significativa anche tra le esportazioni ed il numero di addetti totali. La presenza di 
alcune variabili esplicative tra loro dipendenti potrebbe creare problemi nella stima del modello di regressione logistica; per questo motivo, per ciascuna regressione logistica è stata verificata la presenza di multicollinearità tra le variabili indipendenti (come illustrato più sotto)

Al fine di stabilire se le evidenze emerse dalle precedenti tabelle sono statisticamente significative, sono stati stimati 7 differenti modelli di regressione logistica (si veda la Tab. 6).

Tab. 6: Stime delle regressioni logistiche

\begin{tabular}{|c|c|c|c|c|c|c|c|c|c|c|c|}
\hline & M1 & M2 & M3 & M4 & M5 & M6 & M7 & M8 & M9 & M10 & M11 \\
\hline Dimensione del Comune & -0.111 & & & & & & -0.018 & 0.117 & -0.027 & -0.149 & 0.133 \\
\hline Comune litoraneo & & -0.216 & & & & & -0.206 & $-0.410^{*}$ & -0.123 & 0.021 & -0.068 \\
\hline \multicolumn{12}{|l|}{ Provincia (rif: Ancona) } \\
\hline Pesaro Urbino & & & 0.261 & & & & & 0.339 & 0.209 & 0.234 & -0.133 \\
\hline Macerata & & & -0.270 & & & & & -0.345 & $-0.628^{* *}$ & -0.445 & $-0.589^{*}$ \\
\hline Fermo & & & $0.558^{*}$ & & & & & $0.602^{*}$ & $0.629^{*}$ & $0.653^{*}$ & 0.741 \\
\hline Ascoli Piceno & & & 0.301 & & & & & 0.309 & 0.422 & 0.488 & $0.772^{* *}$ \\
\hline Addetti Totali & & & & $0.156^{* \star *}$ & & & & & $0.167^{* \star \star}$ & $0.108^{\star \star \star *}$ & $0.112^{* \star *}$ \\
\hline \multicolumn{12}{|l|}{ Export (rif.: Nella Provincia) } \\
\hline Extra Europa & & & & & $2.060^{* \star *}$ & & & & & $1.428^{\star \star \star}$ & 0.632 \\
\hline Nella UE & & & & & $3.136^{* * *}$ & & & & & $2.578^{\star \star \star *}$ & $2.073^{* * *}$ \\
\hline In Italia & & & & & $1.874^{\star \star *}$ & & & & & $1.553^{\star \star \star}$ & $1.289^{* * *}$ \\
\hline Nella Regione & & & & & $0.669^{* \star *}$ & & & & & $0.651^{\star \star}$ & 0.296 \\
\hline \multicolumn{12}{|l|}{ Settore (rif:: Tessile) } \\
\hline Altri settori manifatturieri & & & & & & $0.618^{*}$ & & & & & $0.752^{*}$ \\
\hline Altri servizi & & & & & & $-0.940^{* *}$ & & & & & 0.167 \\
\hline Calzature & & & & & & 0.513 & & & & & $0.846^{*}$ \\
\hline Legno & & & & & & $1.580^{\star \star \star}$ & & & & & $2.239^{\star \star *}$ \\
\hline Meccanica & & & & & & $1.511^{\star \star \star *}$ & & & & & $1.976^{* * *}$ \\
\hline Servizi alle famiglie & & & & & & $-2.096^{* * *}$ & & & & & -0.855 \\
\hline Ampiezza del campione (n) & 599 & 599 & 600 & 598 & 600 & 600 & 599 & 599 & 597 & 597 & 597 \\
\hline P-value (RV) & 0.531 & 0.270 & 0.074 & 0.000 & 0.000 & 0.000 & 0.542 & 0.058 & 0.000 & 0.000 & 0.000 \\
\hline Pseudo $R^{2}$ & 0.000 & 0.002 & 0.012 & 0.103 & 0.148 & 0.167 & 0.002 & 0.016 & 0.126 & 0.201 & 0.299 \\
\hline AIC & 1.245 & 1.243 & 1.243 & 1.122 & 1.074 & 1.057 & 1.247 & 1.242 & 1.111 & 1.032 & .9304 \\
\hline $\mathrm{BIC}$ & -3076 & -3077 & -3070 & -3143 & -3172 & -3173 & -3070 & -3056 & -3117 & -3147 & -3181 \\
\hline VIF & 1 & 1 & 1 & 1 & 1 & 1 & 1.88 & 1.55 & 1.64 & 1.58 & 1.72 \\
\hline
\end{tabular}

Fonte: ns. elaborazioni su dati EBAM

I primi sei modelli analizzano separatamente gli effetti di ciascuna variabile esplicativa: il primo modello (M1) analizza l'effetto della dimensione del comune dove è localizzata l'attività artigiana; il secondo modello (M2) analizza l'effetto della vicinanza alla costa del comune; il terzo modello (M3) considera le differenze fra imprese localizzate in diverse province; il modello M4 studia l'impatto del numero di addetti; il modello M5 analizza gli effetti dell'ampiezza del mercato di vendita dell'impresa; infine il modello M6 analizza le differenze per tipo di settore economico. Per ciascun modello sono stati calcolati gli indicatori di bontà del modello (Akaike Information Criterion - AIC, Bayesian Information Criterion - BIC, e lo pseudo- $\mathrm{R}^{2}$ ) ed è stato effettuato il test del rapporto di verosimiglianza $(\mathrm{RV})$ per verificare la significatività del modello nel suo complesso. Confrontando i primi sei 
modelli di regressione logistica univariata, emerge chiaramente che il modello con pseudo- $\mathrm{R}^{2}$ più alto e con valori di $\mathrm{AIC}$ e $\mathrm{BIC}$ più bassi è il modello M6, il cui regressore è la variabile Export, mentre i modelli basati rispettivamente sulla dimensione del comune e sul comune litoraneo non sono significativi. Inoltre, è stata testata la significatività di ciascuna variabile esplicativa, mediante test basati sulla stima di massima verosimiglianza. Da queste prime analisi emerge che:

(i) né la dimensione del comune, né la sua vicinanza alla costa hanno un impatto significativo sull'utilizzo di Internet;

(ii) la provincia di Fermo ha un'incidenza dell'uso di Internet significativamente superiore rispetto alle altre provincie marchigiane;

(iii) all'aumentare del numero di addetti la probabilità di usare Internet aumenta significativamente;

(iv) le imprese che vendono i loro prodotti al di fuori della provincia (in regione, in Italia, in Europa o al di fuori dell'Europa) usano il web in modo significativamente maggiore, rispetto alle imprese la cui clientela è prevalentemente locale;

(v) nei settori della lavorazione del legno e della meccanica le imprese usufruiscono di Internet in maniera significativamente maggiore rispetto a quelle del settore tessile, mentre quelle che offrono servizi alle famiglie lo usano in modo ridotto.

Nei modelli successivi (M7-M11) si è studiato l'effetto congiunto delle variabili esplicative, con un metodo sequenziale di inserimento di una variabile alla volta.

Per ciascun modello è stata testata la presenza di multicollinearità tra le variabili indipendenti, ricorrendo all'indicatore VIF (Variance Inflation Factor). Valori dell'indice VIF maggiori di 10 rivelano che la variabile indipendente può essere considerata una combinazione lineare delle altre, mentre, in caso di modello con un solo regressore, l'indice VIF vale 1. Tutti i regressori dei modelli considerati presentano valori dell'indicatore di gran lunga inferiori alla soglia critica (il valore massimo è pari a 2.43 per la dimensione del comune nel modello M7), rivelando quindi l'assenza di multicollinearità nei modelli proposti ${ }^{7}$. Nella Tabella 6 si riporta il valore medio dell'indice VIF per ciascun modello.

Nei modelli M7 e M8 si è studiato l'effetto congiunto delle caratteristiche del territorio in cui è situata l'impresa (dimensione del comune, vicinanza alla costa e provincia); le stime ottenute sono analoghe a quelle dei modelli M1 - M3.

Nel modello M9 viene inserita anche la dimensione dell'impresa e nel modello M10 si aggiungono inoltre le sue esportazioni; le stime ottenute sono analoghe a quelle dei modelli M1 - M5.

Infine, il modello M11 è il modello finale completo, che tiene conto dell'effetto congiunto di tutte le variabili esplicative considerate. Tale modello presenta il più basso valore di AIC e di BIC ed il più alto valore di pseudo- $\mathrm{R}^{2}$, rivelandosi così il modello migliore per spiegare l'utilizzo di internet, tra tutti i modelli qui considerati.

7 Per motivi di brevità, i dati non vengono mostrati nel presente articolo, ma sono disponibili su richiesta agli autori. 
Si nota, innanzitutto, come l'impatto di tutte le variabili esplicative sulla variabile target (ossia il segno dei coefficienti) rimanga pressoché costante al variare dei diversi modelli considerati, rivelando la robustezza delle stime.

Ne deriva che, a parità di tutti gli altri regressori, la probabilità di usare Internet non dipende in modo statisticamente significativo né dalla dimensione del comune nel quale le imprese sono localizzate, né dalla loro vicinanza alla costa. Tale risultato persiste in tutti i modelli considerati.

Analizzando la provincia di appartenenza, la situazione cambia leggermente nel modello finale, in cui si inserisce il settore di appartenenza, rispetto ai modelli precedenti: non è più la provincia di Fermo bensì quella di Ascoli Piceno a distinguersi in maniera significativa dalla provincia di Ancona, mentre la provincia di Macerata è significativamente inferiore ad Ancona nell'uso di Internet.

Una spiegazione a tale cambiamento è in parte dovuta al fatto che la maggior parte delle micro imprese fermane si occupa di calzature, e dunque la significatività di tali imprese emerge nella variabile del settore e non in quella della provincia. Dalla tabella 7 si nota infatti come la provincia di Fermo sia fortemente legata al settore calzaturiero. Ciò spiega perché nel modello M11 tale provincia non risulti più significativa, una volta inserito il settore d'attività tra i regressori del modello.

L'ipotesi di ricerca $\mathrm{H}_{2}$ può quindi essere confermata soltanto in parte, con riferimento alla variabile provincia.

La dimensione aziendale mostra nuovamente un chiaro impatto sulla propensione all'utilizzo di Internet: ceteris paribus al crescere del numero di addetti aumenta la probabilità che l'impresa si avvalga di Internet per la propria attività. Ciò conferma la prima ipotesi di ricerca $\left(\mathrm{H}_{1}\right)$.

Tab. 7: Relazione tra Provincia di appartenenza e settore economico dell'impresa

\begin{tabular}{lrrrrrrrr}
\hline Provincia & Tessile & Calzature & \multicolumn{1}{c}{ Legno } & Meccanica & $\begin{array}{c}\text { Servizi alle } \\
\text { famiglie }\end{array}$ & $\begin{array}{c}\text { Altre attività } \\
\text { manifatturiere }\end{array}$ & $\begin{array}{c}\text { Altri } \\
\text { servizi }\end{array}$ & Totale \\
\hline AN & 19.0 & 0.6 & 8.1 & 23.0 & 14.9 & 10.9 & 23.6 & 100.0 \\
& 37.9 & 1.2 & 16.5 & 44.0 & 30.2 & 27.9 & 42.3 & 29.1 \\
\hline AP & 21.8 & 6.4 & 9.0 & 11.5 & 24.4 & 11.5 & 15.4 & 100.0 \\
& 19.5 & 5.9 & 8.2 & 9.9 & 22.1 & 13.2 & 12.4 & 13.0 \\
\hline FM & 10.0 & 62.0 & 0.0 & 14.0 & 0.0 & 0.0 & 14.0 & 100.0 \\
& 5.8 & 36.5 & 0.0 & 7.7 & 0.0 & 0.0 & 7.2 & 8.4 \\
\hline MC & 7.0 & 30.4 & 12.0 & 10.8 & 13.9 & 13.9 & 12.0 & 100.0 \\
& 12.6 & 56.5 & 22.4 & 18.7 & 25.6 & 32.4 & 19.6 & 26.4 \\
\hline PU & 15.1 & 0.0 & 32.4 & 13.0 & 13.7 & 13.0 & 13.0 & 100.0 \\
& 24.1 & 0.0 & 52.9 & 19.8 & 22.1 & 26.5 & 18.6 & 23.2 \\
\hline Totale & 14.5 & 14.2 & 14.2 & 15.2 & 14.4 & 11.4 & 16.2 & 100.0 \\
& 100.0 & 100.0 & 100.0 & 100.0 & 100.0 & 100.0 & 100.0 & 100.0 \\
\hline
\end{tabular}

Fonte: ns. elaborazioni su dati EBAM

Differenze nell'utilizzo di Internet sono dovute inoltre al diverso settore economico nel quale le imprese operano, a conferma della terza ipotesi di ricerca proposta $\left(\mathrm{H}_{3}\right)$. In particolare, la Tab. 6 mostra che il settore di appartenenza (in particolare il settore meccanico, quello inerente alla lavorazione del legno ed il settore calzaturiero) influenza in modo significativo la probabilità di usare la rete, a 
parità di tutti gli altri regressori. In particolare, l'odds (ossia il rapporto tra la probabilità $p$ di usare Internet e la probabilità di non usarlo) delle aziende che lavorano nel settore del legno è pari a 3.6 volte l'odds di chi lavora nel settore tessile, l'odds delle imprese meccaniche è 7.2 maggiore di quello delle imprese tessili, mentre l'odds del settore calzaturificio corrisponde a 2.3 volte quello del settore tessile ${ }^{8}$.

Anche la quarta ipotesi di ricerca è confermata dall'evidenza empirica; le analisi mostrano chiaramente, infatti, che più ampia è la dimensione del mercato di vendita e maggiore è la probabilità di utilizzare Internet. A differenza del modello M10, una volta inserito il settore economico come variabile di controllo, la vendita dei propri beni e servizi nella regione o in Paesi extra europei non risulta più statisticamente significativa. In particolare, 1 'odds delle imprese che vendono in tutta Italia è pari a 3.6 volte l'odds di quelle che operano soltanto nel territorio provinciale, mentre l'odds delle organizzazioni che esportano anche in Europa è pari a 7.9 volte quello delle imprese che vendono solo in provincia.

Nel modello M11 i "Servizi alle famiglie" e "altri servizi" perdono significatività, poiché, come mostra la tabella n.8, sono fortemente dipendenti dal livello di internazionalizzazione. La commercializzazione "nella regione" perde significatività perché dipende fortemente dal settore calzaturificio (che diventa significativo nel modello M11). L'internazionalizzazione "extra Europa" perde significatività perché dipende fortemente dai settori calzaturiero (che diventa significativo nel modello M11), legno e meccanica.

È evidente quindi che esiste una relazione di "dipendenza" tra la variabile "settore economico" e "livello di internazionalizzazione" e tra la prima e la "provincia di appartenenza" dell'impresa.

Per determinare l'ordine di importanza delle variabili esplicative considerate nell'analisi, è necessario confrontare gli indici pseudo- $R^{2}, A I C$ e $B I C$ di ciascun modello di regressione logistica (indicati nella Tabella 6). Quanto più alto risulta il valore dello pseudo- $R^{2}$ e quanto più bassi sono i valori di $A I C$ e $B I C$, tanto migliore sarà il modello. Come si può notare, le variabili che identificano la localizzazione delle imprese artigiane (dimensione e vicinanza alla costa del comune) non hanno un impatto significativo sulla propensione ad utilizzare Internet (in entrambi i modelli il $p$-value del test del rapporto di verosimiglianza è di gran lunga maggiore di ogni livello di significatività accettabile, e lo pseudo- $R^{2}$ è pari a zero). Ai limiti della significatività è la variabile Provincia, per la quale il $p$-value del test del rapporto di verosimiglianza è inferiore al $10 \%$ e lo pseudo- $R^{2}$ è molto basso (pari a 0.012 ). Le variabili più esplicative per spiegare l'adozione di Internet sono (in ordine crescente di importanza): il numero di addetti (pseudo- $R^{2}$ pari a 0.103 , AIC pari a 1.122 e BIC pari a -3143), l'ampiezza del mercato servito (pseudo- $R^{2}$ pari a 0.148 , AIC pari a 1.074 e BIC pari a) -3172 ) ed il settore di attività (pseudo- $R^{2}$ pari a 0.167 , AIC pari a 1.057 e BIC pari a -3173 ).

8 Come riferimento si è scelto il settore tessile poiché è quello che presenta il valore di utilizzo di Internet più vicino al dato medio. 
Tab. 8 : Relazione tra ampiezza del mercato di vendita e settore economico

\begin{tabular}{lrrrrrr}
\hline \multicolumn{1}{c}{ Settore } & $\begin{array}{c}\text { Al più in } \\
\text { provincia }\end{array}$ & $\begin{array}{c}\text { Al più in } \\
\text { regione }\end{array}$ & $\begin{array}{c}\text { Al più in } \\
\text { Italia }\end{array}$ & Europa & $\begin{array}{c}\text { Extra- } \\
\text { Europa }\end{array}$ & Totale \\
\hline Altre attività manifatturieri & 39.71 & 14.71 & 33.82 & 8.82 & 2.94 & 100 \\
& 8.33 & 10.99 & 16.79 & 22.22 & 10 & 11.35 \\
\hline Altri servizi & 79.38 & 13.4 & 7.22 & 0 & 0 & 100 \\
& 23.77 & 14.29 & 5.11 & 0 & 0 & 16.19 \\
\hline Calzature & 45.88 & 31.76 & 11.76 & 4.71 & 5.88 & 100 \\
& 12.04 & 29.67 & 7.3 & 14.81 & 25 & 14.19 \\
\hline Legno e mobile & 37.65 & 16.47 & 30.59 & 7.06 & 8.24 & 100 \\
& 9.88 & 15.38 & 18.98 & 22.22 & 35 & 14.19 \\
\hline Meccanica & 36.26 & 14.29 & 35.16 & 7.69 & 6.59 & 100 \\
& 10.19 & 14.29 & 23.36 & 25.93 & 30 & 15.19 \\
\hline Servizi alle person & 100 & 0 & 0 & 0 & 0 & 100 \\
& 26.54 & 0 & 0 & 0 & 0 & 14.36 \\
\hline Tessile & 34.48 & 16.09 & 44.83 & 4.6 & 0 & 100 \\
& 9.26 & 15.38 & 28.47 & 14.81 & 0 & 14.52 \\
\hline Totale & 54.09 & 15.19 & 22.87 & 4.51 & 3.34 & 100 \\
& 100 & 100 & 100 & 100 & 100 & 100 \\
\hline
\end{tabular}

Fonte: ns. elaborazioni su dati EBAM

\section{Implicazioni e limitazioni dello studio}

Dal punto di vista teorico, l'analisi svolta fornisce un contributo originale al dibattito sull'adozione di Internet da parte delle imprese artigiane. Il primo risultato che emerge è un limitato utilizzo degli strumenti online da parte delle microimprese: soltanto il 31,7\% del campione ha dichiarato infatti di impiegare Internet nei propri processi gestionali. Il tessuto artigianale marchigiano appare essere contraddistinto da una "modernità incompiuta", nel senso che non ha ancora saputo cogliere a pieno le potenzialità che Internet può offrire. Dal punto di vista delle implicazioni manageriali, si tratta di un'opportunità mancata significativa: diverse ricerche hanno dimostrato che l'utilizzo di Internet può contribuire allo sviluppo internazionale delle imprese, specialmente di quelle di minore dimensione, in quanto aiuta a superare i principali ostacoli che queste possono incontrare, nel momento in cui decidono di avviare il processo di internazionalizzazione. Il canale elettronico consente infatti di raggiungere i potenziali clienti in ogni parte del mondo, a costi notevolmente inferiori e con investimenti significativamente più bassi, rispetto alle modalità "tradizionali" di esportazione. L'utilizzo di Internet come canale di raccolta di informazioni, consente inoltre di ridurre l'incertezza connessa all'ingresso nei mercati esteri, di individuare i migliori partner con i quali collaborare e di velocizzare i contatti con i clienti esteri. Va inoltre osservato che molte piccole imprese competono sul mercato offrendo un prodotto di nicchia, rispetto al quale è individuabile un "segmento di mercato transnazionale" (Silvestrelli, 2008, p. 24), che si estende cioè su più mercati-Paese; il canale elettronico riesce a servire in modo più efficace ed efficiente tale tipologia di segmento, consentendo di entrare velocemente e contemporaneamente in più mercati esteri. 
Il problema è che le imprese più piccole mancano sovente di una visione strategica, di un orientamento al mercato, e non sono consapevoli di quali siano i processi maggiormente influenti sulla propria competitività; ciò si traduce inevitabilmente in una maggiore difficoltà a cogliere le opportunità, soprattutto nei processi di marketing, derivanti dall'innovazione, che in alcuni casi viene vista addirittura come un'inutile complicazione delle attività gestionali svolte quotidianamente. Tale situazione impedisce di fatto agli imprenditori di percepire $\mathrm{i}$ vantaggi derivanti dall'introduzione di Internet e quindi di investire in quella direzione. Appaiono quindi necessari interventi volti ad aumentare "l'absorptive capacity" delle micro-imprese, intesa come capacità di identificare le opportunità di innovazione e di farle proprie, anche mediante iniziative di supporto "mirate" a questo particolare segmento di utenti.

Riguardo ai fattori influenti sul ricorso ad Internet, i risultati ai quali il presente studio è pervenuto confermano alcune evidenze già discusse nella letteratura nazionale ed internazionale per il più ampio comparto delle PMI. Va tuttavia rilevato che le micro imprese risultano, per diversi aspetti gestionali, non omogenee rispetto a questo macro raggruppamento e ciò viene riscontrato anche in questa analisi.

Il ricorso ad Internet non è uniforme nei settori economici indagati: esso è più frequente nei settori manifatturieri, rispetto a quelli dei servizi. Tale risultato appare in parte sorprendente: era infatti abbastanza ragionevole attendersi un maggior uso di Internet nei settori consumer, come quello dei servizi alla persona, rispetto ai settori business to business, come sono la maggior parte di quelli manifatturieri inclusi nello studio. Una possibile spiegazione potrebbe essere fornita dal fatto che l'adozione di Internet da parte delle micro-imprese industriali sia stata indotta da "pressioni" provenienti dalle imprese clienti di maggiore dimensione, già utilizzatrici di queste tecnologie. Non sono poche infatti le evidenze empiriche, che mostrano come la decisione di introdurre Internet sia sovente dovuta a stimoli esterni, costituiti proprio dalle pressioni di clienti e di fornitori (Fillis e Wagner, 2005; Caldeira e Ward, 2003; Poon, 2000). All'interno dei settori industriali poi sono emerse differenze significative tra il tessile, il calzaturiero e le "altre attività manifatturiere", da un lato, e il legno e la meccanica, dall'altro, con questi ultimi maggiormente propensi all'adozione di Internet. Un altro aspetto che può influire sui risultati complessivamente analizzati è legato al differenziale dimensionale fra le imprese di servizi e quelle manifatturiere (di dimensioni medie maggiori).

Conformemente ai risultati ottenuti da altri studi, anche nella presente analisi la dimensione aziendale, misurata dal numero di addetti, costituisce un fattore significativo nel determinare l'adozione di Internet nei processi di management, anche se non è risultato quello più influente. Ne deriva che anche all'interno del gruppo delle micro-imprese, si possono riscontrare differenze nell'adozione di Internet ad ulteriore conferma di quel digital divide tra le PMI, evidenziato da Arbore e Ordanini (2006).

Sempre in merito alle evidenze empiriche è possibile osservare che la localizzazione delle imprese, analizzata sotto il profilo della dimensione del comune 
di appartenenza e dell'area geografica (comune costiero - comune dell'entroterra), risulti non influente nell'adozione di Internet nei processi di management e ciò è solo in parte in linea con i risultati di altri studi; va tuttavia rilevato che nella nostra analisi non sono stati isolati i grandi centri urbani rispetto al resto dei comuni e che nella regione non esistono "aree metropolitane". Queste ultime potrebbero evidenziare un tessuto socio-economico differente e pertanto essere maggiormente influente sui fattori presi in considerazione nel presente studio.

La non significatività della localizzazione unita alla significatività della dimensione aziendale, potrebbe essere interpretata inoltre come maggiore rilevanza delle risorse interne all'impresa rispetto alle risorse ambientali esterne nel determinare l'adozione di Internet.

Un ultimo aspetto esaminato, che risulta peraltro di particolare interesse per la nostra analisi, è quello relativo alla possibile influenza che il livello di apertura verso i mercati esteri può esercitare sull'adozione di strumenti web based per lo sviluppo delle attività artigianali; va rilevato in tal senso che, come evidenziato in letteratura, questo fattore può essere considerato sia un "antecedente", sia una conseguenza del ricorso a tali tecnologie. In questa prima fase della ricerca, è stato analizzato come possibile fattore influente sull'adozione e l'evidenza empirica ha fornito una conferma "forte" in tal senso: le imprese che presentano una maggiore propensione a vendere oltre i confini italiani sono quelle che, con maggiore probabilità, adottano Internet. Una possibile spiegazione di ciò potrebbe essere data dal fatto che le imprese maggiormente internazionalizzate presentano una complessità informativa $\mathrm{e}$ gestionale superiore rispetto alle altre, tale da incentivare l'impiego di Internet nei propri processi gestionali.

Come già evidenziato, i risultati presentati rappresentano la prima parte di un più ampio lavoro di ricerca, che comprende, oltre al livello di adozione, anche lo studio delle modalità secondo le quali le micro imprese e le imprese artigiane utilizzano Internet per la loro attività ed, in particolare, per le politiche di marketing e lo sviluppo delle attività commerciali. È evidente che ciò influisce sulla completezza e sull'approfondimento del lavoro, il quale presenta pertanto i limiti tipici di un progetto work in progress.

Un limite dell'analisi, che potrebbe costituire un'interessante tema di ricerca futura, è il non aver considerato nel presente studio l'influenza della figura dell'imprenditore, delle sue competenze, del profilo professionale, dell'età; non può sfuggire infatti che, nelle imprese minori, il ruolo dell'imprenditore risulta sovente determinante in gran parte delle decisioni strategiche, e quindi anche in quelle relative all'adozione delle nuove tecnologie. Lo sviluppo della micro-impresa, inteso come arricchimento delle competenze e delle abilità, è strettamente interdipendente con lo sviluppo dell'imprenditore. Va anche evidenziato che la variabile dipendente "uso di Internet" non discrimina tra le diverse possibili applicazioni della Rete, né fornisce indicazioni sul grado di utilizzo della Rete stessa.

Un ulteriore tema di studio futuro potrebbe essere costituito dall'analisi delle implicazioni dell'adozione di Internet sulla performance delle micro-imprese. La 
maggior parte degli studi compiuti sulla relazione tra Internet e performance non considerano infatti le specificità di questa categoria.

Non può sfuggire inoltre che le peculiarità delle piccole imprese italiane (sovente appartenenti a distretti industriali, come in diversi casi nel campione esaminato) complica la generalizzabilità dei risultati ottenuti ad altri contesti geografici.

\section{Bibliografia}

ALAM S.S. (2009), "Adoption of Internet in Malaysian SMEs", Journal of Small Business and Enterprise Development, vol. 16, n. 2, pp. 240-255.

ARBORE A., ORDANINI A. (2006), "Broadband divide among SMEs. The role of size, location and outsourcing strategies", International Small Business Journal, vol. 24, n. 1, pp. 83-99.

BAYO-MORIONES A., LERA-LOPEZ F. (2007), "A firm-level analysis of determinants of ICT adoption in Spain”, Technovation, vol. 27, n. 6-7, pp. 352-366.

BENGTSSON M., BOTER H., VANYUSHYN V. (2007), "Integrating the Internet and marketing operations. A study of antecedents in firms of different size", International Small Business Journal, vol. 25, n. 1, pp. 27-48.

BORDONABA-JUSTE V., LUCIA-PALACIO L., POLO-REDONDO Y. (2012), "The influence of organizational factors on e-business use: analysis of firm size", Marketing Intelligence \& Planning, vol. 30, n. 2, pp. 212-229.

BRYNJOLFSSON E. (1993), "The productivity paradox of information technology", Communications of the ACM, vol. 36, n. 12, pp. 66-77.

CALDEIRA M.M., WARD J.M. (2003), "Using resource-based theory to interpret the successful adoption and use of information systems and technology in manufacturing small and medium-sized enterprises", European Journal of Information Systems, vol. 12 , pp. $127-141$.

CEDROLA E. (2009), "Internet per le piccole e medie imprese: potenzialità e stato dell'arte in Italia e in Europa", Mercati e Competitività, n. 1, pp. 20-43.

CENTRO STUDI CNA (2012), Le imprese, nonostante tutto.

CHEUNG W.M., HUANG W. (2002), "An investigation of commercial usage of the World Wide Web: a picture from Singapore", International Journal of Information Management, vol. 22, n. 5, pp. 377-388.

DANDRIDGE T., LEVENBURG N.M. (2000), "High-tech potential? An exploratory study of very small firms' usage of the Internet", International of Small Business Journal, vol. 18, n. 2, pp. 81-91.

DAVIS P.S., HARVESTON P.D. (2000), "Internationalization and organizational growth: the impact of Internet usage and technology involvement among entrepreneur-led family business", Family Business Review, vol. XIII, n. 2, pp. 107-120.

DAVIS C.H., VLADICA F. (2006), "Use of Internet technologies and e-Business solutions: a structural model of sources of business value among Canadian micro-enterprises", Proceedings of the $39^{\text {th }}$ Hawaii International Conference on System Science, Jan. 0407, Kauai, USA.

DHOLAKIA R.R., KSHETRI N. (2004), "Factors impacting the adoption of the Internet among SMEs", Small Business Economics, vol. 23, pp. 311-322. 
DRENNAN J., MCCOLL-KENNEDY J.R. (2003), "The relationship between Internet use and perceived performance in retail and professional service firms", Journal of Service Marketing, vol. 17, n. 3, pp. 295-311.

DREW S. (2003), "Strategic use of e-commerce by SMEs in the East of England", European Management Journal, vol. 21, n. 1, pp. 79-88.

FILLIS I., JOHANNSON U., WAGNER B. (2004), "Factors impacting on e-business adoption and development in the smaller firm", International Journal of Entrepreneurial Behavior \& Research, vol. 10, n. 3, pp. 178-191.

FILLIS I., WAGNER B. (2005) "E-business Development: An Exploratory Investigation of the Small Firm”, International Small Business Journal, vol. 23, n.6, pp. 604-634.

GOLINELLI G. (2009), "L'approccio sistemico-vitale: nuovi orizzonti di ricerca per il governo dell'impresa", Sinergie, n. 79, pp. XI-XXIV.

GOLINELLI G., GATTI M. (2007), "Il pensiero di Roberto Fazzi e l'approccio sistemico al governo dell'impresa: spunti di riflessione tra debito culturale, avanzamento dottrinale e spirito del tempo", Sinergie, n. 72, pp. 207-255.

GRANDON E.E., PEARSON J.M. (2004), "Electronic commerce adoption: an empirical study of small and medium US business", Information and Management, vol. 42, n. 1, pp. 197-216.

GREGORI G.L., CARDINALI S., TEMPERINI V. (2012), Traiettorie di sviluppo delle imprese calzaturiere nel nuovo contesto competitivo, Giappichelli, Torino.

HAIRUDDIN H., NOR LAILA MD. N., ARIFF MD AB M. (2012), "Why do microenterprise refuse to use information technology: a case of Batik microenterprises in Malaysia", in Procedia - Social and Behavioral Sciences, International Conference on Asia Pacific Business Innovation and Technology Management, vol. 57, pp. 494502.

HOLLENSTEIN H. (2004), "Determinants of the adoption of information and communication technologies. An empirical analysis based on firm-level data for the Swiss business sector", Structural Change and Economic Dynamics, vol. 15, n. 3, pp. 315-342.

IHLSTROM C., NILSSON M. (2003), "E-business adoption by SMEs - prerequisites and attitudes of SMEs in a Swedish network", Journal of Organizational Computing and Electronic Commerce, vol. 13, n. 3/4, pp. 211-223.

JOHNSTON D.A., WADE M., MCCLEAN R. (2007), "Does e-Business matter to SMEs? A comparison of the financial impacts of Internet business solutions on Europe and North American SMEs", Journal of Small Business Management, vol. 45, n. 3, pp. 354-361.

JUTLA D., BODORIK P., DHALIWAL J. (2002), "Supporting the e-business readiness of small and medium-sized enterprises: approaches and metrics", Internet Research: Electronic networking applications and policy, vol. 12, n. 2, pp. 139-164.

KHEMTHONG S., ROBERTS L.M. (2006), "Adoption of Internet and web technology for hotel marketing: a study of hotels in Thailand", Journal of Business Systems, Governance and Ethics, vol. 1, n. 2, pp. 47-60.

KOWTHA N.R., CHOON T.W. (2001), "Determinants of website development: a study of electronic commerce in Singapore", Information and Management, vol. 39, n. 3, pp. 227-242.

LERTWONGSATIEN C., WONGPINUNWATANA N. (2003). "E-commerce adoption in Thailand: An empirical study of Small and Medium Enterprises (SMEs)." Journal of Global Information Technology Management, vol. 6, n. 3, pp. 67-83. 
LEVENBURG N.M. (2005), "Does size matters? Small firms' use of e-business tools in the supply chain", Electronic Markets, vol. 15, n. 2, pp. 94-105.

LEVY M., POWELL P., WORRALL L. (2005), "Strategic Intent and E-business in SMEs: enablers and inhibitors", Information Resources Management Journal, vol. 18, n. 4, pp. 1-20.

LOVE P.E.D., IRANI Z., STANDING C., LIN C., BURN J.M. (2005), “The enigma of evaluation: benefits, costs and risks of IT in Australian small-medium-sized enterprises", Information \& Management, vol. 42, n. 1, pp. 947-964.

MOSTAFA R.H.A., WHEELER C., JONES M.V. (2005), "Entrepreneurial orientation, commitment to the Internet and export performance in small and medium sized exporting firms”, Journal of International Entrepreneurship, vol. 3, n. 4, pp. 291-302.

PARKER C.M., CASTLEMAN T. (2007), "New directions for research on SME-eBusiness: insights from an analysis of journal articles from 2003 to 2006", Journal of Information Systems and Small Business, vol. 1, n. 1-2, pp. 21-40.

PASCUCCI F. (2012), "Il web, il marketing e la crisi: prime riflessioni su alcuni casi aziendali” in Franco M., Gregori G.L., Marcone M.R. (a cura di), Le opportunità oltre la crisi. Prospettive manageriali e strategie pubbliche dei Paesi dell'Europa del Sud, Società Editrice Esculapio, Bologna, pp. 171-192.

PELTIER J.W., ZHAO Y., SCHIBROWSKU J.A. (2012), "Technology adoption by small business: an exploratory study of the interrelationships of owner and environmental factors", International Small Business Journal, vol. 30, n. 4, pp. 406-431.

PONTIKAKIS D., LIN Y., DEMIRBAS D. (2006), "History matters in Greece: the adoption of Internet-enabled computers by small and medium sized enterprises", Information Economics and Policy, vol. 18, n. 3, pp. 332-358.

POON S. (2000), "Business environment and Internet commerce benefits - Small business perspective", European Journal of Information Systems, vol. 9, n. 2, pp. 72-81.

POON S., SWATMAN P.M.C. (1999), "An exploratory study of small business Internet commerce issues", Information \& Management, vol. 35, n. 1, pp. 9-18.

PREMKUMAR G., RAMAMURTHY D. (1995), "The role of interorganizational and organizational factors on the decision mode for adoption on interorganizational systems", Decision Sciences, vol. 26, n. 3, p. 303-336.

PREMKUMAR, G. AND ROBERTS, M. (1999). "Adoption of New Information Technologies in Rural Small Businesses. Omega: The International Journal of Management Science, vol. 27, pp 467-484.

SILVESTRELLI S. (2008), International Marketing Mix, Società Editrice Esculapio, Bologna.

TAN K.S., CHONG S.C., LIN B., EZE U.C. (2010), "Internet-based ICT adoption among SMES: demographic versus benefits, barriers, and adoption intention.", Journal of Enterprise Information Management, vol. 23, n. 1, pp. 27-55.

THE BOSTON CONSULTING GROUP (2011), Fattore Internet. Come Internet sta trasformando l'economia italiana.

THONG J.Y.L., YAP C.S. (1995), "CEO characteristics, organizational characteristics and information technology adoption in small business", Omega, vol. 23, n. 4, pp. 429442.

VESCOVI T., IESEPPON G. (2002), "L'evoluzione di Internet come strumento di comunicazione e di marketing nelle imprese minori", Micro \& Macro Marketing, anno XI, n. 3, pp. 509-521. 
WADE M., JOHNSTON D., MCCLEAN R. (2004), "Exploring the net impact of internet business solution adoption on SME performance", International Journal of Electronic Business, vol. 2, n. 4, pp. 336-350.

WALCZUCH R., VAN BRAVEN G., LUNDGREN H. (2000), "Internet adoption barriers for small firms in the Netherlands", European Management Journal, vol. 18, n. 5, pp. 561-572. 
